\title{
Identification of a novel cDC2-committed progenitor within mouse common dendritic cell progenitor population
}

\section{Dear Editor,}

Dendritic cells (DCs) are the most efficient professional antigen presenting cells that act as sentinels of the immune system and conduct vital functions in the initiation and regulation of innate and adaptive immunities (Wculek et al., 2020). In addition to being critical cellular components in pathogen clearance, DCs are promising targets for improved tumor immunotherapy, vaccine design, and intervention of autoimmune diseases (Nutt and Chopin, 2020). DCs can be classified as plasmacytoid DCs (pDCs) and conventional DCs (cDCs) in both mouse and human (Anderson et al., 2018; Nutt and Chopin, 2020). The cDCs comprise two developmentally and functionally distinct subsets known as $\mathrm{CD} \mathrm{a}^{+}$(also $\mathrm{CD} 24^{+} / \mathrm{XCR} 1^{+} / \mathrm{CD} 103^{+}$) $\mathrm{cDC} 1$ and $\mathrm{CD} 11 \mathrm{~b}^{+}$ (also SIRPa ${ }^{+}$) CDC2 in mouse (Anderson et al., 2018; Nutt and Chopin, 2020). cDC1 are specialized in antigen crosspresentation and activation of cytotoxic $T$ lymphocytes that are crucial effectors of cellular immunity. While cDC2 are featured as antigen presenting cells of extracellular pathogens and promoting various helper $\mathrm{T}$ cell differentiation, including Th2 and Th17, therefore playing essential roles in humoral and cellular immunity (Nutt and Chopin, 2020).

Given that each DC subset performs unique and irreplaceable functions in immune responses, it underpins that DC development and differentiation are indispensable in modulating DC-centric immune responses. All DC subsets can arise from $\mathrm{Lin}^{-} \mathrm{C}-\mathrm{Kit}^{\text {int }} \mathrm{Flt}{ }^{+} \mathrm{CD} 11 \mathrm{c}^{-} \mathrm{IL}-7 \mathrm{R}^{-}$common DC progenitors (CDPs) identified within bone marrow (BM) (Naik et al., 2007; Onai et al., 2007, 2013). CDPs can be divided into two subfractions according to the expression level of CD115. The CD115 $\mathrm{CDPs}$ are enriched for precursors with pDC differentiation potential, while CD $115^{+}$CDPs preferably produce more cDCs (Naik et al., 2007; Onai et al., 2007, 2013). Recent studies suggest that $C D 115^{+}$CDPs are heterogeneous and already exhibit the transcriptional priming of the $\mathrm{CDC} 1$ or $\mathrm{CDC} 2$ lineage, leading to separate differentiation into pre-cDC1 and pre-cDC2 (Schlitzer et al., 2015). Indeed, Zeb2 ${ }^{\text {lo }}$ and Id $2^{\text {hi }}$ CDPs prone to produce cDC1 (Bagadia et al., 2019). However, the existence of CDC2-primed progenitors within CDPs remains to be identified. In this study, we identified a $\mathrm{Ly} 6 \mathrm{C}^{+}$subset amongst $\mathrm{CD}_{115^{+}} \mathrm{CDPs}$, representing $\mathrm{CDC} 2$-commited progenitors.

As expression of Ly6C served as a lineage marker for distinguishing pre-cDC2, it may also be implicated in $\mathrm{CDC} 2$ priming during the CDP stage (Schlitzer et al., 2015; Dress et al., 2019). We therefore examined the cell-surface expression of Ly6C on CDPs and found that Ly6C was only expressed by a fraction of CD $115^{+} \mathrm{CDPs}$, but not by CD $115^{-}$ CDPs. Ly6C segregated CDPs into three subsets, namely CD115 Ly6C $^{-},{\text {CD } 115^{+}{\text {Ly } 6 C^{-}}^{-} \text {, and CD } 115^{+} \text {Ly6C }}^{+}$CDPs (Fig. 1A). The abundance of CD115 ${ }^{+}$Ly6 $C^{+}$CDPs accounted for approximately $25 \%$ of $\mathrm{CD} 115^{+}$CDPs and $10 \%$ of total CDPs (Fig. 1B and 1C). Furthermore, the surface protein

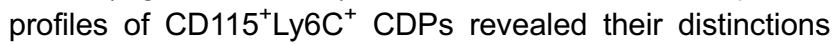
from any other CD115- or Ly6C-expressing BM progenitors, including $\mathrm{Lin}^{-} \mathrm{C}-\mathrm{Kit}^{+} \mathrm{Flt} \mathrm{H}^{+} \mathrm{CD} 115^{+} \mathrm{CD} 11 \mathrm{~b}^{-} \mathrm{Ly} 6 \mathrm{C}^{-}$macrophage DC progenitors (MDPs), Lin $^{-} \mathrm{C}-\mathrm{Kit}^{+} \mathrm{Flt3} 3^{-} \mathrm{CD} 115^{+} \mathrm{CD} 11-$ $\mathrm{b}^{-} \mathrm{Ly}_{6 \mathrm{C}^{+}}$common monocyte progenitors (cMoPs), and c-Kit ${ }^{-} \mathrm{Flt} 3^{-} \mathrm{CD} 115^{+} \mathrm{CD} 11 \mathrm{~b}^{+}$Ly6C $\mathrm{C}^{\text {lo }}$ or Ly6C $^{\text {hi }}$ monocytes (Figs. 1D and S1A).

To assess the differentiation potentials of and relationships amongst $\mathrm{CD} 115^{-}$Ly $\mathrm{C}^{-}, \mathrm{CD}^{-} 115^{+} \mathrm{Ly}_{6 \mathrm{C}^{-}}$, and CD $115^{+}$Ly6 $6 C^{+}$CDP subsets, each CDP subset was purified and cultured by in vitro $\mathrm{DC}$ differentiation system in the presence of Flt3L (Naik et al., 2005). Both CD $115^{-}$Ly $_{6 C^{-}}$and CD $115^{+} \mathrm{Ly}_{6 C^{-}}$CDPs could give rise to CD $115^{+} \mathrm{Ly}_{6 C^{+}} \mathrm{CDP}$ progenies at day 1 (Fig. S1B), indicating the relative downstream position of $C D 115^{+}$Ly6C $C^{+}$CDPs among the three CDP subsets. Furthermore, the differentiation potentials of the three CDP subsets were determined by analyzing the composition of their progenies in culture on day 3 and 6 .

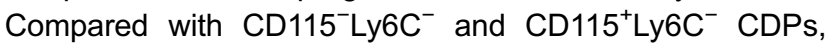

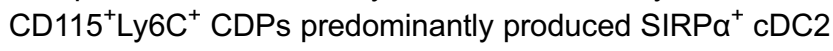
on day 3 , the time to obtain maximum amounts of $C D C$ products (Figs. $1 \mathrm{E}-\mathrm{G}$ and $\mathrm{S} 2 \mathrm{~A}$ ), implying their commitment toward the $\mathrm{CDC} 2$ lineage. Moreover, the CD115 ${ }^{+} \mathrm{Ly} 6 \mathrm{C}^{+}$ CDPs displayed lower proliferative capacity than that of the other two CDP subsets (Fig. S2A). Consequently, these results suggested that the $\mathrm{CD} 115^{+} \mathrm{Ly} 6 \mathrm{C}^{+} \mathrm{CDPs}$ gave rise 
A

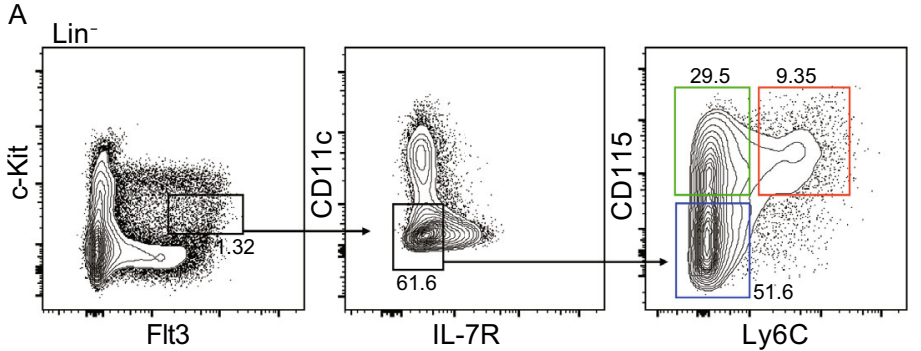

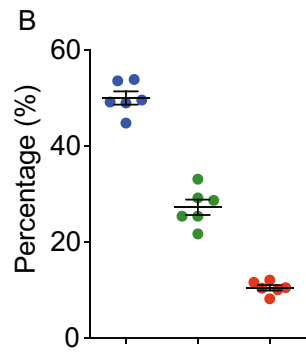

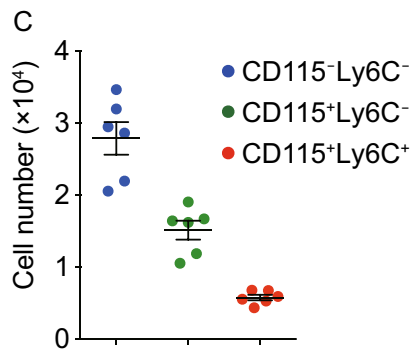

D
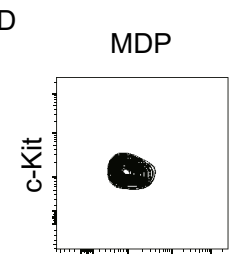
cMoP
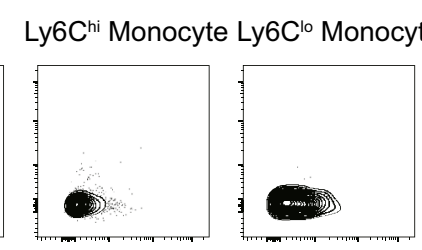

CD115-Ly6C CDP

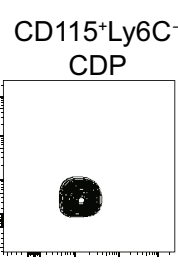
CD115+Ly6C+
CDP
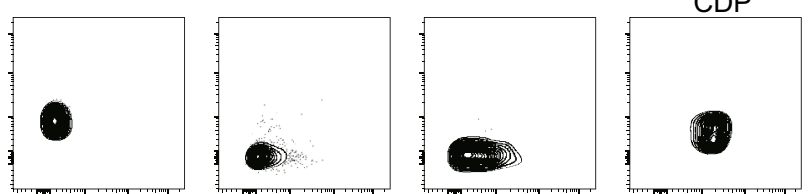
FIt3
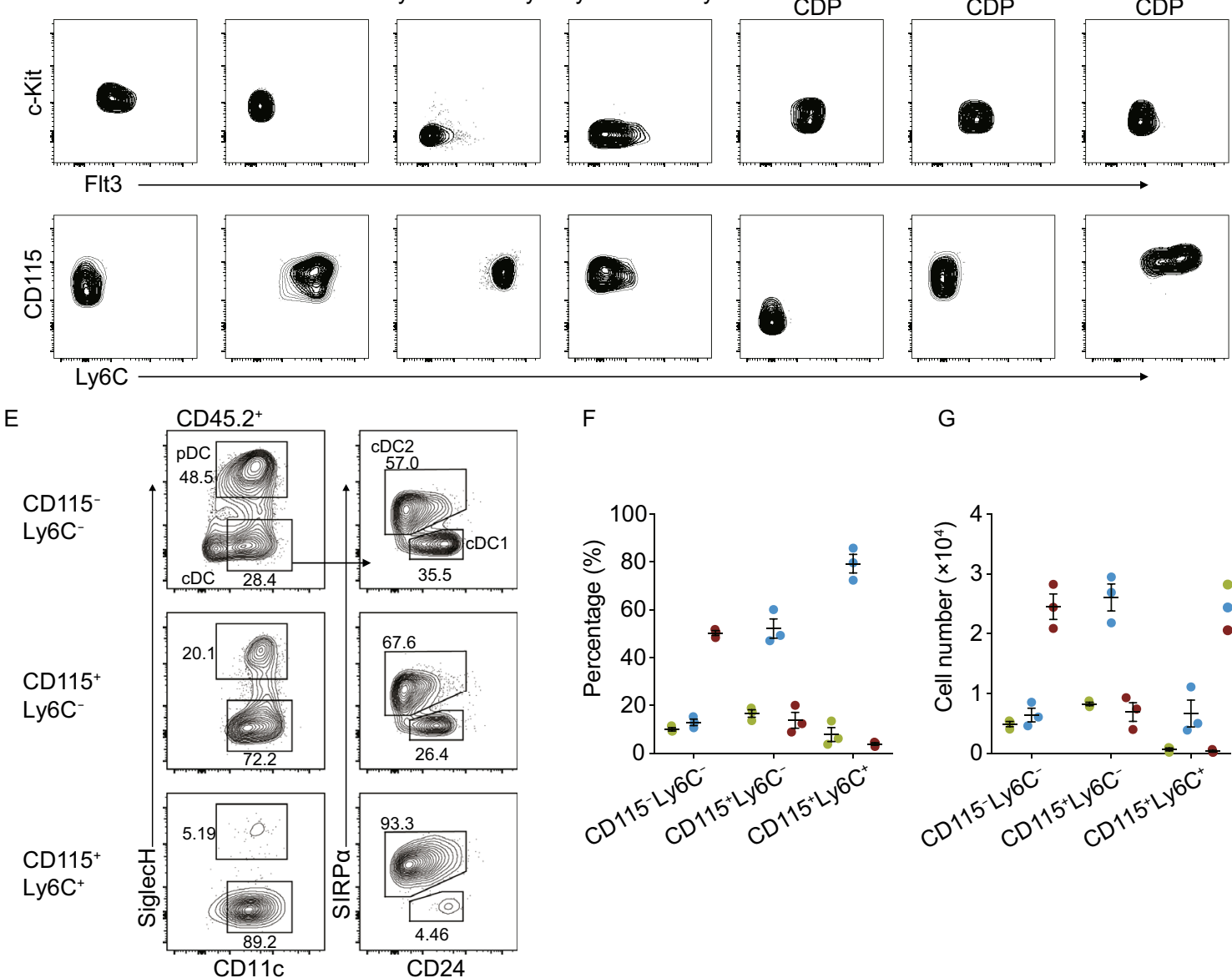

F

G
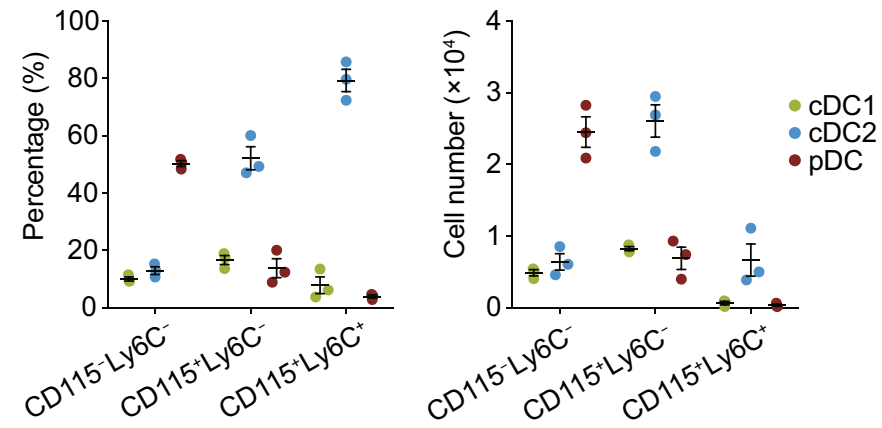

Figure 1. Identification of $c D C 2$-primed $C D 115^{+} L y 6 C^{+}$subset within CDPs. (A) Flow cytometry analysis of the expression of

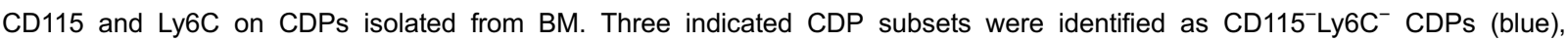
CD115 ${ }^{+}$Ly6C $C^{-}$CDPs (green), and CD115 ${ }^{+}$Ly6C $C^{+}$CDPs (red). (B) Percentages within total CDPs and (C) cell numbers of three indicated CDP subsets defined as in (A) $(n=6)$. (D) Flow cytometry analysis of the expression of Flt3, c-Kit, CD115, and Ly6C on macrophage DC progenitors (MDPs), common monocyte progenitors (cMoPs), Ly6 $\mathrm{C}^{\text {hi }}$ monocytes, Ly6 $\mathrm{C}^{\mathrm{lo}}$ monocytes, and three indicated CDP subsets. (E-G) Purified CD45.2 ${ }^{+}$CD $115^{-}{\text {Ly } 6 C^{-}, \text {CD } 115^{+} \text {Ly6C }}^{-}$, and CD $115^{+}$Ly6C ${ }^{+}$CDPs were co-cultured with $\mathrm{CD} 45.1^{+}$total BM cells in the medium containing Flt3L $(100 \mathrm{ng} / \mathrm{mL})$, respectively. (E) Representative flow-cytometric profiles, (F) percentages, and $(G)$ cell numbers of $C D 45.2^{+}$DC subsets derived from indicated CDP subsets at day $3(n=3)$. Data in $(D-G)$ are representative of three independent experiments. Data in $(B, C, F$, and $G)$ are represented as mean \pm SEM.

mainly to $\mathrm{cDC} 2$, with developmentally more mature properties compared to CD115 Ly6C $^{-}$and CD $115^{+}$Ly6C $^{-}$CDPs.

To further confirm the developmental potentials of the three CDP subsets in vivo, we performed adoptive transfer of the three CDP subsets. At 10 days post transfer, the numbers of progenies derived from the three subsets of CDP all peaked in the recipient spleen (Fig. S2B). Consistent with the results of in vitro cultures, CD115 ${ }^{+}$Ly6C ${ }^{+}$CDPs 
generated predominantly $\mathrm{CDC} 2$, but little $\mathrm{pDC}$ and $\mathrm{CDC} 1$ subsets in the spleen (Fig. 2A-C). Flow cytometry analysis demonstrated that, $\mathrm{CDC} 2$ derived from $\mathrm{CD} 115^{+} \mathrm{Ly}_{6 \mathrm{C}^{-}}$and

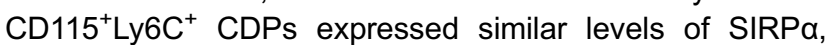
CD11b, ESAM, and CD4, while CDC2 derived from CD115 $^{-}$Ly6C $^{-}$CDPs showed lower ESAM and CD4 expression (Fig. 2D). In accordance with previous investigations (Onai et al., 2013), the CD115 ${ }^{-}$Ly6C $^{-}$CDPs produced abundant pDCs in recipient spleen and BM, whereas $\mathrm{CD}_{115^{+} \mathrm{Ly}_{6 C}}^{-}$and CD115 ${ }^{+} \mathrm{Ly}_{6 C^{+}}$CDPs produced only a few and no pDCs respectively (Figs. $2 \mathrm{~A}-\mathrm{C}, \mathrm{S} 2 \mathrm{C}$, and S2D). As expected, all three CDP subsets did not generate T cells, B cells, NK cells, or other myeloid cells in recipient spleen and BM (Fig. S3), reconfirming their DC-restricted differentiation capacity. Moreover, CD $115^{+}{\text {Ly } 6 C^{+}}^{+}$CDPs also generated predominately $\mathrm{CDC} 2$ in nonlymphoid tissues, including the small intestine and lung (Fig. S4). Altogether, these data demonstrated that $C D 115^{+} \mathrm{Ly}_{6 C^{+}}$CDPs represent a $\mathrm{CDC} 2-$ committed progenitor subset within CDPs.

In order to determine the differences among the three CDP subpopulations at molecular level, we compared the transcriptional signatures by RNA sequencing analysis (Fig. S5). CD115 ${ }^{+}$Ly6C ${ }^{+}$CDPs expressed higher levels of maturation-associated genes, including Cst3, Fcer1g, Crip1, Ifi30, Anxa2, and Anxa5 (Schlitzer et al., 2015), consistent with their more differentiated features (Fig. 2E). Meanwhile, cDC2 signature genes, including Sirpa, Clec10a, Tyrobp, Fcer1g, Lyz2, Csf1r, and Klf4 (Schlitzer et al., 2015), were

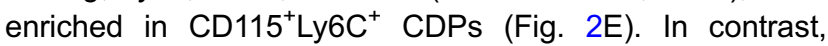

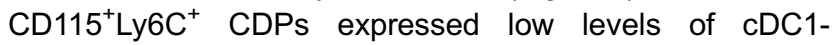

associated Id 2 transcript and minimal levels of pDC-associated transcripts Siglech, Ly6d, Tcf4, and Tsc22d1 (Schlitzer et al., 2015; Dress et al., 2019; Nutt and Chopin, 2020), further validating their cDC2-restricted differentiation potential (Fig. 2E). In addition, all three CDP subsets did not express Itgax (encoding CD11c), a marker expressed by pre-DCs and mature DCs, indicating that they were at a developmental stage earlier than pre-DCs (Fig. 2E). Furthermore, in terms of expression of key transcription factors involved in $\mathrm{DC}$ development, in comparison with pre-cDC2, CD $115^{+}$Ly6C $^{+}$CDPs expressed higher levels of Irf8 than that of pre-cDC2, but similar to that of the other two CDP subsets (Fig. 2E and 2F). Whereas the expression levels of genes associated with cDC2 differentiation Irf4, KIf4, and Zeb2 were upregulated in pre-cDC2, confirming that pre-cDC2 were at a later stage than CD $115^{+} \mathrm{Ly}_{6 \mathrm{C}^{+}} \mathrm{CDPs}$ during $\mathrm{CDC} 2$ differentiation. As expected, both CD $115^{+}{\text {Ly } 6 C^{+} \text {CDPs and }}^{+}$ pre-cDC2 expressed low levels of Id2, a cDC1 associated gene (Fig. 2F). Thus, these results demonstrated that

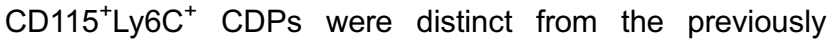
defined CD11 ${ }^{+}$pre-cDC2 (Schlitzer et al., 2015).

Taken together, we identified CD115 ${ }^{+}$Ly6C ${ }^{+}$CDPs as a novel cDC2-committed progenitor subset within CDP population, and demonstrated that the commitment to CDC2 lineage occurred at the CDP stage earlier than pre-DC stage. Our findings provide novel insights into the lineage commitment of $\mathrm{CDC} 2$, and the CD $115^{+} \mathrm{Ly}_{6 C^{+}}$CDPs may serve as a potential target for modulating $\mathrm{CDC} 2$ differentiation and function, which will facilitate further explorations of cDC2mediated immune modulations and therapies. 
A

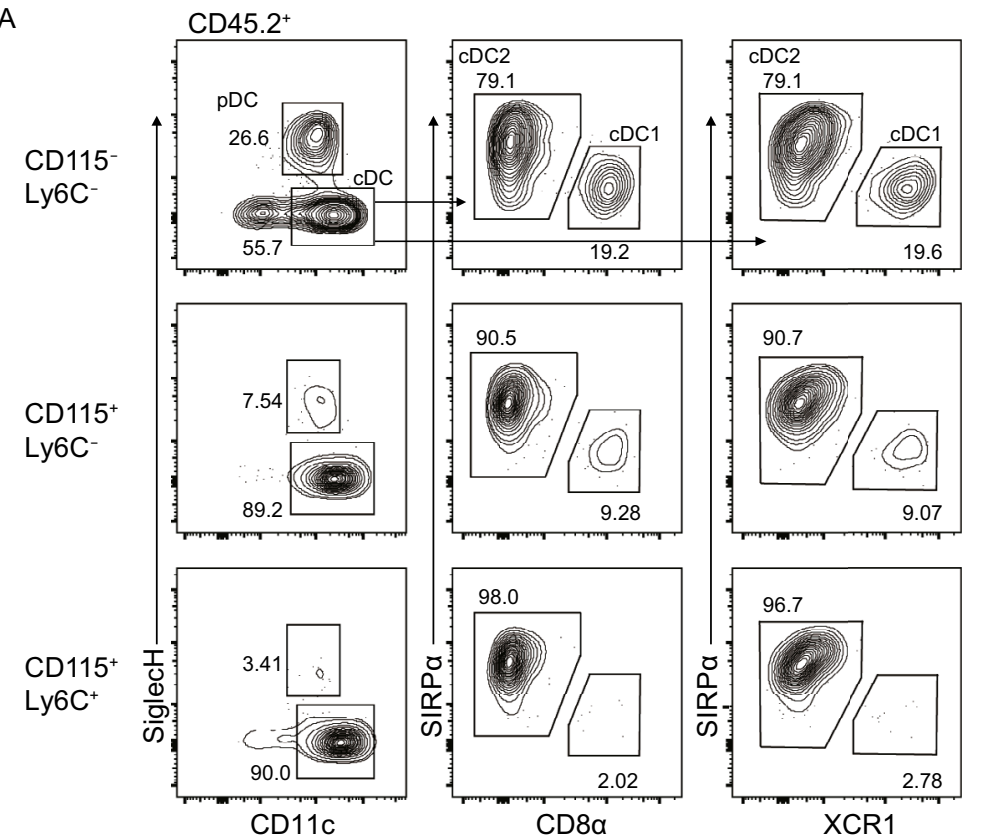

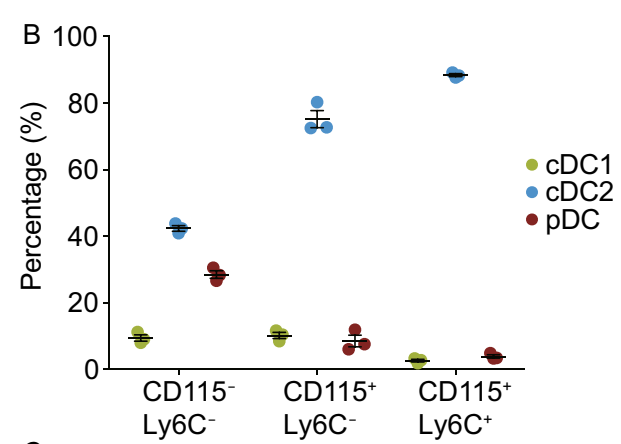

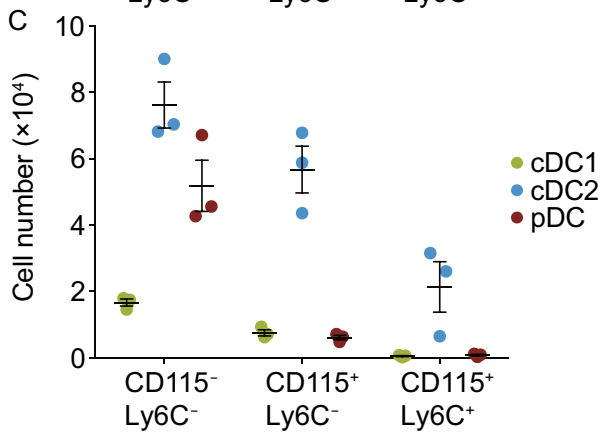

。
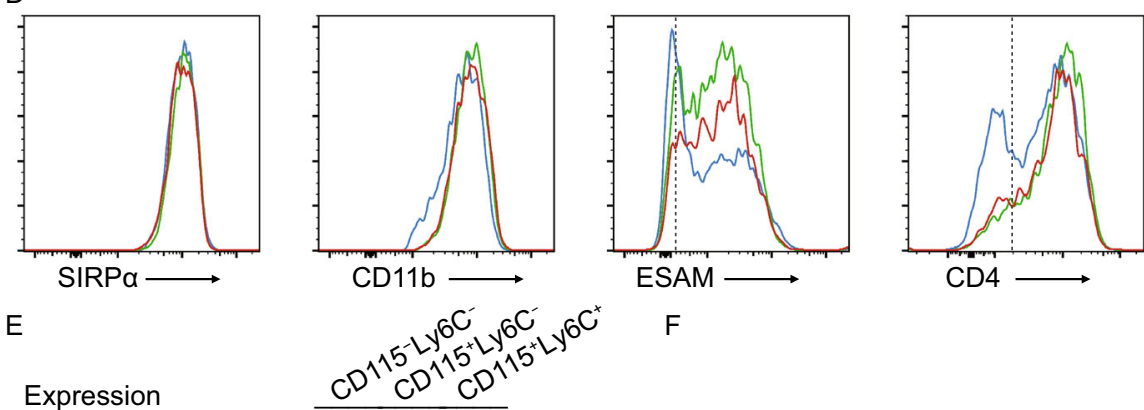

- CD115-Ly6C- CDP-derived CDC2

- CD115+Ly6C- CDP-derived CDC2

$-\mathrm{CD} 115^{+} \mathrm{Ly}_{6 \mathrm{C}}+\mathrm{CDP}$-derived CDC2 
4 Figure 2. In vivo differentiation potential and characterization of cDC2-primed CD115 ${ }^{+}$Ly $6 C^{+}$CDPs. (A-C) Purified

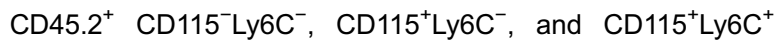
CDPs $\left(2 \times 10^{4}\right)$ were transplanted with CD45. $1^{+}$BM cells $(2 \times$ $10^{5}$ ) into irradiated $C D 45.1^{+}$recipient mice via intravenous injection. (A) Representative flow-cytometric profiles, (B) percentages, and $(C)$ cell numbers of splenic CD45.2 ${ }^{+}$DC subsets 10 days post-transplantation. (D) Expression of ESAM and CD4 on gated donor-derived $\mathrm{CD} 11 \mathrm{c}^{+} \mathrm{SIRPa}{ }^{+} \mathrm{CD} 11 \mathrm{~b}^{+} \mathrm{CDC} 2$ cells. Dashed lines indicate positive staining threshold. (E) $\log _{2^{-}}$ transformed (FPKM + 1) expression values of selected genes encoding products associated with $\mathrm{DC}$ development, assessed in CD115 Ly6C $^{-}$, CD115 ${ }^{+}$Ly6C $^{-}$, and CD115 ${ }^{+}$Ly6C ${ }^{+}$CDPs $(n=$ 2). Left margin, hierarchical clustering. (F) Expression of selected genes on purified CD $115^{+}$Ly6C ${ }^{+}$CDPs and preCDC2 were measured by qRT-PCR and represented by arbitrary units relative to Actb $(n=3 \sim 5)$. Data in (A-D) are representative of three independent experiments $(n=3)$. Data in $(B, C$, and $F)$ are represented as mean \pm SEM.

\section{FOOTNOTES}

This research was supported by the Ministry of Science and Technology of People's Republic of China (National Key R\&D Program 2019YFA0508502 to L. Wu), National Natural Science Foundation of China (grants 31991174 and 91642207 to L. Wu), and TsinghuaPeking Center for Life Sciences (L. Wu).

The authors have no relevant financial or non-financial interests to disclose.

The use and care of animals complied with the guideline of the Institutional Animal Care and Use Committee at Tsinghua University.

All data generated or analysed during this study are included in this published article and its supplementary information files.

$Y$. Tian and X. Guo designed research, conducted experiments, analyzed the data. Y. Tian, X. Guo, T. Wu, and K. Fei wrote the manuscript. L. Wu conceptualized, designed, supervised the project, and wrote the manuscript. All authors revised and approved the manuscript.

\footnotetext{
Yujie $\operatorname{Tian}^{1,2,3}$ (D), Xueheng Guo ${ }^{1,3,4}$ (D), Tao $\mathrm{Wu}^{1,3}$ (D), Kuangyu $\mathrm{Fei}^{1}$ (D), $\mathrm{Li} \mathrm{Wu}^{1,3 凶}$ (D)

${ }^{1}$ Institute for Immunology, Tsinghua-Peking Center for Life Sciences, School of Medicine, Tsinghua University, Beijing 100084, China

2 Joint Graduate Program of Peking-Tsinghua-National Institute of Biological Sciences, School of Life Sciences, Tsinghua University, Beijing 100084, China

${ }^{3}$ Beijing Key Laboratory for Immunological Research on Chronic Diseases, Beijing 100084, China
}

4 Present address: National Education Examinations Authority, Beijing 100084, China

$\bowtie$ Correspondence: wuli@tsinghua.edu.cn (L. Wu)

\section{OPEN ACCESS}

This article is licensed under a Creative Commons Attribution 4.0 International License, which permits use, sharing, adaptation, distribution and reproduction in any medium or format, as long as you give appropriate credit to the original author(s) and the source, provide a link to the Creative Commons licence, and indicate if changes were made. The images or other third party material in this article are included in the article's Creative Commons licence, unless indicated otherwise in a credit line to the material. If material is not included in the article's Creative Commons licence and your intended use is not permitted by statutory regulation or exceeds the permitted use, you will need to obtain permission directly from the copyright holder. To view a copy of this licence, visit http:// creativecommons.org/licenses/by/4.0/.

\section{REFERENCES}

Anderson DA, Murphy KM, Briseño CG (2018) Development, diversity, and function of dendritic cells in mouse and human. Cold Spring Harb Perspect Biol 10:a028613

Bagadia P, Huang X, Liu T-T, Durai V, Grajales-Reyes GE, Nitschke M, Modrusan Z, Granja JM, Satpathy AT, Briseño CG et al (2019) An Nfil3-Zeb2-Id2 pathway imposes Irf8 enhancer switching during $\mathrm{CDC} 1$ development. Nat Immunol 20:1174-1185

Dress RJ, Dutertre CA, Giladi A, Schlitzer A, Low I, Shadan NB, Tay A, Lum J, Kairi MF, Hwang YY et al (2019) Plasmacytoid dendritic cells develop from Ly6D+ lymphoid progenitors distinct from the myeloid lineage. Nat Immunol 20:852-864

Naik SH, Proietto Al, Wilson NS, Dakic A, Schnorrer P, Fuchsberger M, Lahoud MH, O'Keeffe M, Shao QX, Chen WF et al (2005) Cutting edge: generation of splenic CD8+ and CD8- dendritic cell equivalents in Fms-like tyrosine kinase 3 ligand bone marrow cultures. J Immunol 174:6592-6597

Naik SH, Sathe P, Park HY, Dakic A, Schnorrer P, Fuchsberger M, Lahoud MH, O'Keeffe M, Shao QX, Chen WF et al (2007) Development of plasmacytoid and conventional dendritic cell subtypes from single precursor cells derived in vitro and in vivo. Nat Immunol 8:1217-1226

Nutt SL, Chopin M (2020) Transcriptional networks driving dendritic cell differentiation and function. Immunity 52:942-956

Onai N, Obata-Onai A, Schmid MA, Ohteki T, Jarrossay D, Manz MG (2007) Identification of clonogenic common Flt3+M-CSFR+ plasmacytoid and conventional dendritic cell progenitors in mouse bone marrow. Nat Immunol 8:1207-1216 
Onai N, Kurabayashi K, Hosoi-Amaike M, Toyama-Sorimachi N, Matsushima K, Inaba K, Ohteki T (2013) A clonogenic progenitor with prominent plasmacytoid dendritic cell developmental potential. Immunity 38:943-957

Schlitzer A, Sivakamasundari V, Chen J, Sumatoh HR, Schreuder J, Lum J, Malleret B, Zhang S, Larbi A, Zolezzi F et al (2015) Identification of $\mathrm{CDC} 1$ - and $\mathrm{CDC} 2$-committed $\mathrm{DC}$ progenitors reveals early lineage priming at the common DC progenitor stage in the bone marrow. Nat Immunol 16:718-728

Wculek SK, Cueto FJ, Mujal AM, Melero I, Krummel MF, Sancho D (2020) Dendritic cells in cancer immunology and immunotherapy. Nat Rev Immunol 20:7-24 